\title{
LOW LEVEL LASER THERAPY (LLLT) FOR CHRONIC JOINT PAIN OF THE ELBOW, WRIST AND FINGERS
}

\author{
Ikuko Okuni, ${ }^{1}$ Nobuyuki Ushigome, ${ }^{1}$ Takashi Harada, ${ }^{1}$ \\ Toshio Ohshiro, ${ }^{2}$ Yoshiro Musya ${ }^{3}$ and Masayuki Sekiguchi ${ }^{4}$ \\ 1: Department of Rehabilitation, Toho University School of Medicine, Tokyo \\ 2: Japan Medical Laser Laboratory, Shinjuku, Tokyo \\ 3: Department of Orthopaedic Surgery, Toho University School of Medicine, \\ Ohashi Hospital, Tokyo \\ 4: Department of Orthopaedic Surgery, Toho University School of Medicine, Tokyo
}

\begin{abstract}
Background and Aims: In previous studies, we successfully applied Low Level Laser Therapy (LLLT) in patients with non-specific chronic pain of the shoulder joint and lower back. The purpose of the present study was to assess the effectiveness of LLLT for chronic joint pain of the elbow, wrist, and fingers.

Subjects and Methods: Nine male and 15 female patients with chronic joint pain of the elbow, wrist, or fingers, who were treated at the rehabilitation outpatient clinic at our hospital from April, 2007 to March, 2009 were enrolled in the study. We used a $1000 \mathrm{~mW}$ semiconductor laser device. Each tender point and three points around it were irradiated with laser energy. Each point was irradiated twice for $20 \mathrm{~s}$ per treatment, giving a total of three minutes for all 4 points. Patients visited the clinic twice a week, and were evaluated after four weeks of treatment. Pain was evaluated with a Visual Analogue Scale (VAS). Statistical analysis of the VAS scores after laser irradiation was performed with Wilcoxon's signed rank sum test, using SPSS Ver.17.

Results: All VAS scores were totaled and statistically analyzed. The average VAS score before irradiation was $59.2 \pm 12.9$, and $33.1 \pm 12.2$ after the irradiation, showing a significant improvement in VAS score $(\mathrm{p}<0.001)$ after treatment. The treatment effect lasted for about one and a half days in the case of wrist pain, epicondylitis lateralis (tennis elbow), and carpal tunnel syndrome. In other pain entities, it lasted for about three to fifteen hours. No change in the range of motion (ROM) was seen in any of the 24 subjects.
\end{abstract}

Conclusion: We concluded that LLLT at the wavelength and parameters used in the present study was effective for chronic pain of the elbow, wrist, and fingers.

Key Words: Low Level Laser Therapy; Elbow, Wrist and Finger Pain Patients; Chronic Pain

\section{Introduction}

The three biggest features unique to human beings are bipedalism, language, and use of hands, but it is the hands that are considered equivalent to a second brain.

Addressee for Correspondence:

Takashi Harada, MD

Dept. of Rehabilitation Medicine

Toho University School of Medicine

6-11-1 Omori-nishi, Ota-ku

Tokyo 143-8541 Japan

Tel: +81-3-3762-4151 / Fax: +81-3-3768-6117

E-mail: haradat_3522@yahoo.co.jp
Hands enable humans to grasp, write, and to collaborate with the eyes in order to control specific skilled motor activities. This differentiates human beings from other animals. Therefore, hand disabilities interfere with activities of daily living (ADL). Many patients seek treatment for chronic pain of the elbow joint, wrist, or fingers and visit the outpatient clinic for rehabilitation at our hospital. With the purpose of pain attenuation for these patients, we administered laser treatment

Manuscript received: September 16th, 2011

Accepted for publication: December 14th, 2011

J-STAGE advance published: December 31st, 2011 
(Low Level Laser Therapy: LLLT) and gave daily life guidance. The goal of this investigation was to examine the efficacy of our treatment.

\section{Subjects and Methods}

\section{Subjects:}

The study subjects comprised 24 patients suffering from chronic pain of the elbow, wrist, or fingers who visited the rehabilitation department of our hospital between April, 2007 and March, 2009. Nine of them were male, and fifteen were female (Table 1). The ages ranged from 26 to 85, with an average of 55.3 years.

Chronic wrist joint torsion was seen in 5 patients; epicondylitis lateralis (tennis elbow) in 7; carpal tunnel syndrome in 4; thumb CM arthropathy in 4; and thumb tendovaginitis in 4 . The patients taking anti-inflamma-

Table 1: CaseOutlines

\begin{tabular}{cccccc}
\hline \multirow{2}{*}{ Case } & \multirow{2}{*}{ Age } & \multirow{2}{*}{ Sex } & \multirow{2}{*}{ Diagnosis $\left(^{* *}\right)$} & \multicolumn{2}{c}{ Pain Score $\left(^{*}\right)$} \\
& & & Pre & Post \\
\hline 1 & 52 & M & W & 80 & 50 \\
2 & 67 & M & W & 80 & 30 \\
3 & 26 & F & W & 40 & 10 \\
4 & 61 & F & W & 50 & 25 \\
5 & 53 & F & W & 40 & 40 \\
6 & 78 & M & E & 60 & 35 \\
7 & 65 & F & E & 60 & 30 \\
8 & 49 & M & E & 70 & 45 \\
9 & 48 & F & E & 70 & 40 \\
10 & 52 & F & E & 40 & 10 \\
11 & 47 & M & E & 60 & 30 \\
12 & 57 & M & E & 50 & 20 \\
13 & 42 & M & C & 70 & 20 \\
14 & 38 & F & C & 60 & 40 \\
15 & 58 & F & C & 60 & 30 \\
16 & 44 & F & C & 60 & 20 \\
17 & 39 & F & O & 40 & 20 \\
18 & 78 & M & O & 60 & 40 \\
19 & 47 & M & O & 75 & 40 \\
20 & 63 & F & O & 70 & 55 \\
21 & 85 & F & S & 40 & 35 \\
22 & 78 & F & S & 70 & 45 \\
23 & 33 & F & S & 50 & 35 \\
24 & 67 & F & S & 65 & 30 \\
\hline
\end{tabular}

*VAS: Visual Analogue Scale

** W: Wrist joint pain

E: Elbow epicondylitis

C: Carpal Tunnel syndrome

$\mathrm{O}$ : Osteoarthritis of the thumb CM joint

S: Snapping thumb tory drug were told to maintain their dose during the whole process of LLLT. Cases after plastic surgery were excluded. After having had the study explained to them in full, all patients gave written informed consent to participate in the study, and the study was approved by the Ethics Committee of Toho University School of Medicine.

\section{Methods:}

We used a semiconductor therapeutic laser device (MDL-2001, Matsushita Electric Corporation, Tokyo, Japan) delivering $1000 \mathrm{~mW}$ in continuous wave at a nominal wavelength of $830 \mathrm{~nm}$ (Figure1), with all the specifications as shown in Table 2. LLLT was delivered by a registered physical therapist. The LLLT treatment

Table 2: Low Level Laser Therapy device specifications

\begin{tabular}{ll}
\hline Laser Element & $\begin{array}{l}\text { Semiconductor Laser Diode } \\
\text { Ga-Al-AS : Gallium-Aluminum-arsenide }\end{array}$ \\
Model \& & $\begin{array}{l}\text { MDL-2001 model } \\
\text { Manufacturer }\end{array}$ \\
Wavelength & $\begin{array}{l}\text { Matshita Electric Corporation, Tokyo, Japan } \\
\text { Wat } \pm 15 \mathrm{~nm}\end{array}$ \\
Output & $\begin{array}{l}1000 \mathrm{~mW} \pm 20 \% \\
\text { continuous wave mode } \\
\text { Mode }\end{array}$ \\
contact mode with positive pressure \\
Irradiated area & Diameter, $14 \mathrm{~mm}$; irradiated area, $1.5 \mathrm{~cm}^{2}$ \\
Irradiation time & $30 \mathrm{sec}$ \\
Energy density & $\underline{20.1 \mathrm{~J} / \mathrm{cm}^{2}}$ \\
Power supply & $100 \mathrm{VAC}, 50-60 \mathrm{~Hz}$ \\
\hline
\end{tabular}

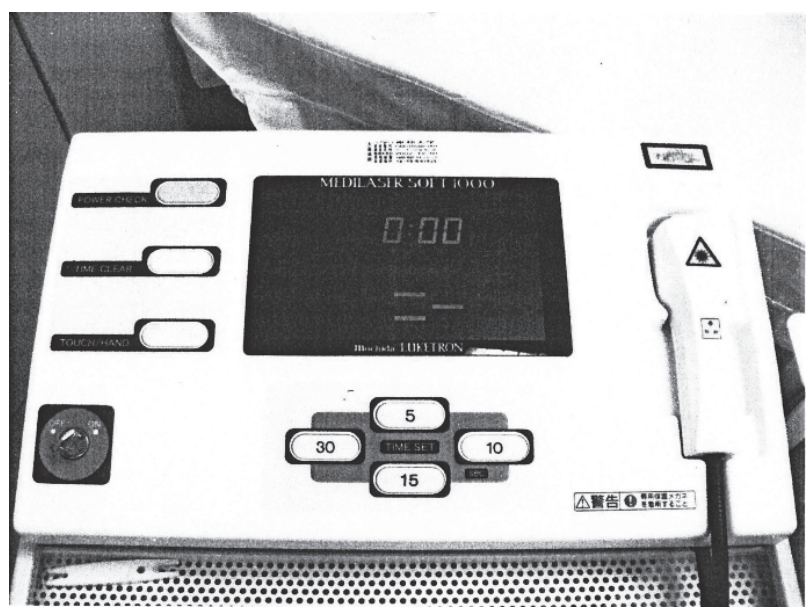

Fig. 1: The LLLT device used in the present study showing the controls, display and the handpiece on the left. 

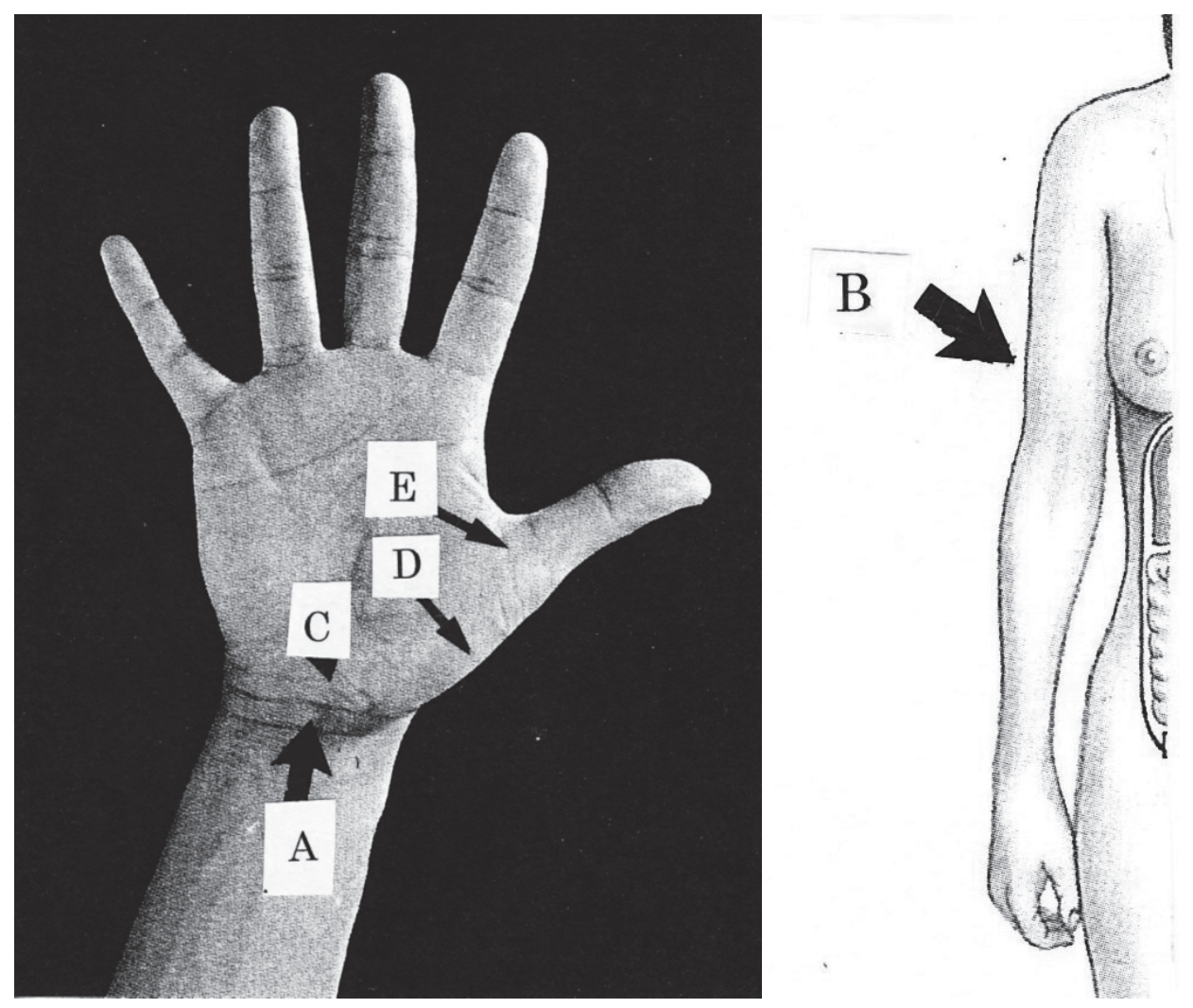

Figure2: Treatment targets \& pain entities
A: Wrist joint
B: Elbow epicondylitis
C: Carpal tunnel syndrome
D: Thumb CM osteoarthritis
E: Snapping thumb

targets were the tender points and three parts around the tender points (Figure 2) with two treatments of thirty seconds each. The incident power density per point was approximately $650 \mathrm{~mW} / \mathrm{cm}^{2}$, delivering an energy density per point per irradiation of $20.1 \mathrm{~J} / \mathrm{cm}^{2}$. We evaluated the results after four weeks of treatment. A Visual Analogue Scale (VAS) was applied to evaluate the pain.

As for range of motion (ROM), the angle of flexion and extension were measured before and after irradiation. ROM was evaluated right after the irradiation. Regarding daily life guidance, we suggested that the subjects should avoid postures that easily cause or worsen the pain, taking their work and daily living environments into consideration. Statistical analysis of the VAS scores before and after laser irradiation was performed with Wilcoxon's signed rank sum test, using SPSS Ver.17.

\section{Results}

Five patients with wrist pain had an average VAS score before treatment of 58 and after treatment of 37 . The latent period of the treatment effect was 31.2 hours on average. Seven patients with pain caused by epicondylitis lateralis (tennis elbow) had an average VAS result of 59 before treatment and 30 after treatment, with an average effect latency of 36.9 hours. Four patients with carpal tunnel syndrome had an average VAS result of 63 before treatment and 28 after treatment, with an average effect latency of 30 hours. Four patients with thumb CM arthropathy had an average VAS result of 61 before treatment and 39 after treatment, with an average latency of 15.8 hours. Four patients with thumb tendovaginitis had an average VAS result of 56 before treatment and 36 after treatment, with an average latency of 3.3 hours. Duration time, in 
other words, the latency of the treatment effect in the case of wrist pain, epicondylitis lateralis (tennis elbow), and carpal tunnel syndrome was about one and a half days, and the others ranged from three to fifteen hours. No change in the range of motion between baseline and after treatment was noted in any of the 24 subjects. The VAS scores were statistically analyzed. The average VAS scores before irradiation were $59.2 \pm 12.9$ and $33.1 \pm 12.2$ after irradiation, showing a significant improvement $(\mathrm{p}<0.001)$. No side effects were found.

\section{Discussion}

We considered that LLLT would be effective from the elbow joint to the forearm, wrist, and fingers, since these parts have less soft tissue which enables the laser to reach the affected part easily, especially given the deep penetrating properties of $830 \mathrm{~nm}$. We have seen many studies on LLLT treatment of the upper limbs; ${ }^{1-5}$ ) however, there are only a few reports about activities of daily living (ADL), range of motion, postures to ease pain, and daily life guidance. $\left.{ }^{6}\right)$

Flexion and extension of the elbow joint enable us to reach objects. General symptoms of patients whose chief complaints are acute or chronic pain around the elbow joint are caused by epicondylitis lateralis (tennis elbow), joint mouse and joint sprain, when we exclude bone fractures and tumors. Among the causes of pain related to forearm pronation and supination are epicondylitis lateralis (tennis elbow) and radius or ulna bony dysplasia.

We often see outpatients with fatigue pain of the forearm muscles due to clerical work. Flexion and extension of the elbow joint, as well as forearm pronation and supination are related to the strain of carrying heavy baggage. With elbow, wrist, and finger pain, it is important to observe the relationship between the unique function of each joint and ADL-movement in relation to the patient's occupation. In the case of wrist pain, there have been many reports about carpal tunnel syndrome. ${ }^{4,6-8)}$ In addition, there are cases of Koendeck's disease, wrist sprains, and so on.

Some serious diseases which cause finger pain are arthropathy of the $\mathrm{CM}$ joint, ${ }^{3,9)}$ and rheumatoid arthritis of the MP joint and PIP joints. 5) We often see flexor tendon and tendovaginitis as causes of wrist pain on the volar site. When patients have DIP joint deformation and pain, we often see osteoarthritis as a main factor.

As for evidence based medicine (EBM), and the body's reaction to LLLT; Akai ${ }^{10)}$ emphasized the necessity of standardizing specifications, such as wavelength, irradiation time, and other parameters before discussing the effectiveness of LLLT results. Regarding EBM of LLLT, however, many experimental results have been reported. ${ }^{11-17)}$ It is, however, difficult to standardize LLLT specifications as strictly as has been suggested in actual clinical practice, due to large interindividual differences among both patients and conditions.

In the present study, we applied LLLT in patients who had no previous plastic surgery. We considered that it may be easier for the laser to reach the painful spot on the elbow, wrist, or finger due to less fatty tissue in these areas. Therefore, we believe that LLLT on the elbow, wrist, and finger can achieve a better beneficial effect than in other parts with more fatty tissue. Elbows, wrists, and fingers; however, are frequently used throughout the day, so treatment duration was limited to four to six hours. To make the treatment duration longer, we have to be aware of the importance of daily life guidance:

1 to avoid the postures which easily cause or exacerbate pain,

2 to think about better ways to use elbows, wrists, and fingers at work,

3 to take breaks when working with the hands.

The VAS score of Case 5 did not change before and after the irradiation, which means that LLLT was ineffective in this patient. We assumed that this is because the patient was a guitarist and thus continuously used his fingers while playing the guitar, thus exerting high levels of stress, negating the beneficial effects of LLLT.

The latency of the treatment effect varied from one hour to forty-eight hours, with an average of twenty-six hours. Regarding any relationship between the latency of the treatment effect and individual patient characteristics, we could not see any special trend of treatment effect with any disorders. Therefore, we assumed that the treatment effect could depend on the frequency of the use of joints during normal ADL.

\section{Conclusion}

We applied LLLT sessions for chronic pain of the elbow, wrist, and fingers, and concluded that it was effective, based on our evaluation of the VAS score. On the other hand, the ROM score did not change after irradiation. 


\section{References}

1: Kenichi Wachi (2009). Low Level Laser Therapy for elbow epicondylitis. J of Japan Laser Therapy Association 2:22-26.

2: Neslihan Ozkan and Lale Altan (2004). Investigation of the Supplementary Effect of GaAs Laser Therapy on the Rehabilitation of Human Digital Flexor Tendons. J of Clinical Laser Medicine Surgery 22:105-110.

3: Brosseau L and Wells G (2005). Randomized controlled trial on low level laser therapy in the treatment of osteoarthritis of the hand. Laser Surg Med 36:210-219.

4: Kenneth Brance and Margaret A Naeser (1999). Carpal Tunnel Syndrome: Clinical Outcome After Low Level Laser Acupuncture. J of Alternative Complementary Medicine 5:5-26.

5: Nury Handikin and Tiur Farida IS (2005). Effects of low power laser on hand rheumatoid arthritis. J Japanese Laser Association (Abstract) 26:147.

6: Wong E, Lee G, Zucherman J and Masen DT (1995). Successful management of female office workers with "repetitive stress injury" or "carpal tunnel syndrome" by a new treatment modality--application of low level laser. International Journal of Clinical Pharmacology and Therapeutics 33:208211.

7: Sunderland S (1976). The nerve lesion in carpal tunnel syndrome. J Neurol Neurosurg Psychiatry 39:615-626.

8: Vennix MJ,Hirsh DD, Faye YCT (1998). Predicting acute denervation in carpal tunnel syndrome. Arch Phys Med Rehabil 78:306-312.

9: Jih MH, Goldberg LH, Kimyai Asadi A (2008). Fractional photothermolysis for photoaging of hands. Dermatol Surg 34:73-78.

10: Masami Akai (2006). Efficacy of Low Level Laser Therapy in View of Evidence Based Medicine. J of Japan Laser Therapy Association 5:20-24

11: Kenji Yoshida (2007). Low Level Laser Therapy for Neurological Disease of Oral Medicine. J of Japan Laser Medicine 28:77-83.

12: Noriko Satou (2003). The efficacy of photo therapy on the arthritis of adjuvant rat: an experimental study. Chubu Seisai Journal 46:179-186.

13: Soichiro Hasimoto (2007). Efficacy of Low Level Laser Therapy for Pain Attenuation. J of Japan Laser Medicine 28:58-67.

14: Rochkind S, El Ani D, Nevo Z, Shahar A (2009). Increase of neuronal sprouting migration using 780 $\mathrm{nm}$ laser phototherapy as procedure for cell therapy. Lasers Sur Med 41:277-281.

15: Enwemeka CS, Cohen Kornberg E, Duswalt EP (1994). Biomechanical effects of three different periods of GASA laser photostimulation on tenotomized tendons. Laser Therapy 6:181-188.

16: Clip CM, Jarow JP, Friend NM (2009). Noninvasive Laser vasectomy. Laser Surg Med 41:203-207.

17: Brondon P,S tadler I, Lanzafame RJ (2009). Pulsing influences photoradiation outcome in cell culture. Lasers Surg Med 41:222-226. 